\title{
Effect of Lumbar Stabilization and Dynamic Lumbar Strengthening Exercises in Patients With Chronic Low Back Pain
}

\author{
Hye Jin Moon, MD, Kyoung Hyo Choi, MD, Dae Ha Kim, MD, Ha Jeong Kim, MD, \\ Young Ki Cho, Kwang Hee Lee, Jung Hoo Kim, Yoo Jung Choi
}

Department of Rehabilitation Medicine, Asan Medical Center, University of Ulsan College of Medicine, Seoul, Korea

\begin{abstract}
Objective To compare the effects of lumbar stabilization exercises and lumbar dynamic strengthening exercises on the maximal isometric strength of the lumbar extensors, pain severity and functional disability in patients with chronic low back pain (LBP).

Methods Patients suffering nonspecific LBP for more than 3 months were included prospectively and randomized into lumbar stabilization exercise group $(\mathrm{n}=11)$ or lumbar dynamic strengthening exercise group $(\mathrm{n}=10)$. Exercises were performed for 1 hour, twice weekly, for 8 weeks. The strength of the lumbar extensors was measured at various angles ranging from $0^{\circ}$ to $72^{\circ}$ at intervals of $12^{\circ}$, using a MedX. The visual analog scale (VAS) and the Oswestry Low Back Pain Disability Questionnaire (ODQ) were used to measure the severity of LBP and functional disability before and after the exercise.

Results Compared with the baseline, lumbar extension strength at all angles improved significantly in both groups after 8 weeks. The improvements were significantly greater in the lumbar stabilization exercise group at $0^{\circ}$ and $12^{\circ}$ of lumbar flexion. VAS decreased significantly after treatment; however, the changes were not significantly different between the groups. ODQ scores improved significantly in the stabilization exercise group only.

Conclusion Both lumbar stabilization and dynamic strengthening exercise strengthened the lumbar extensors and reduced LBP. However, the lumbar stabilization exercise was more effective in lumbar extensor strengthening and functional improvement in patients with nonspecific chronic LBP.
\end{abstract}

Keywords Exercise, Low back pain, Muscle strength, Abdominal muscle, Rehabilitation

Received June 29, 2012; Accepted August 29, 2012

Corresponding author: Kyoung Hyo Choi

Department of Rehabilitation Medicine, Asan Medical Center, University of Ulsan College of Medicine, 88 Olympic-ro 43-gil, Songpa-gu, Seoul 138-736, Korea

Tel: +82-2-3010-3800, Fax: +82-2-3010-6964, E-mail: khchoi@amc.seoul. $\mathrm{kr}$

() This is an open-access article distributed under the terms of the Creative Commons Attribution Non-Commercial License (http://creativecommons. org/licenses/by-nc/3.0) which permits unrestricted noncommercial use, distribution, and reproduction in any medium, provided the original work is properly cited.

Copyright $\odot 2013$ by Korean Academy of Rehabilitation Medicine

\section{INTRODUCTION}

Chronic low back pain (LBP) defined as back pain lasting more than 12 weeks, affects over $50 \%$ of the general population [1]. It is estimated that over $70 \%$ of the adults have at least one episode of LBP in their lifetime [2]. Because the causes of LBP are variable, different exercise regimens have been used to treat patients, including lumbar flexion, extension, isometric flexion, passive extension, and intensive dynamic back exercise regimens 
[3-6]. Many of these exercise regimes have not yielded satisfactory results $[7,8]$, although it is accepted that some form of exercise is better than none $[9,10]$.

Recently, there has been a focus on exercises that aim to maintain/improve lumbar spine stability [11]. Although no formal definition of lumbar stabilization exercises exists, the approach is aimed at improving the neuromuscular control, strength, and endurance of the muscles that are central to maintaining the dynamic spinal and trunk stability. Several groups of muscles particularly targeted the transversus abdominis and lumbar multifidi, but also other paraspinal, abdominal, diaphragmatic, and pelvic muscles [12].

Unsubstantiated suggestions that stabilization training may be useful in reducing pain and disability for all patients with nonspecific LBP, have appeared in the litera- ture [13], but these assertions have not been definitively demonstrated. The overwhelming majority of studies on lumbar stabilization exercises use mixed groups (including patients with disc lesions, osteoarthritis, or leg pain) of subjects with nonspecific chronic LBP [12]. These studies cannot determine whether a specific subgroup of patients may be more responsive to lumbar stabilization exercises. Also, no randomized controlled trials have measured the maximal isometric contraction strength of lumbar extensors at different angles of lumbar flexion. Therefore, the aim of this study was to compare the effects of lumbar stabilization exercises and lumbar dynamic strengthening exercises on the maximal isometric contraction strength of the lumbar extensors, pain severity, and functional disability in patients with nonspecific chronic LBP.

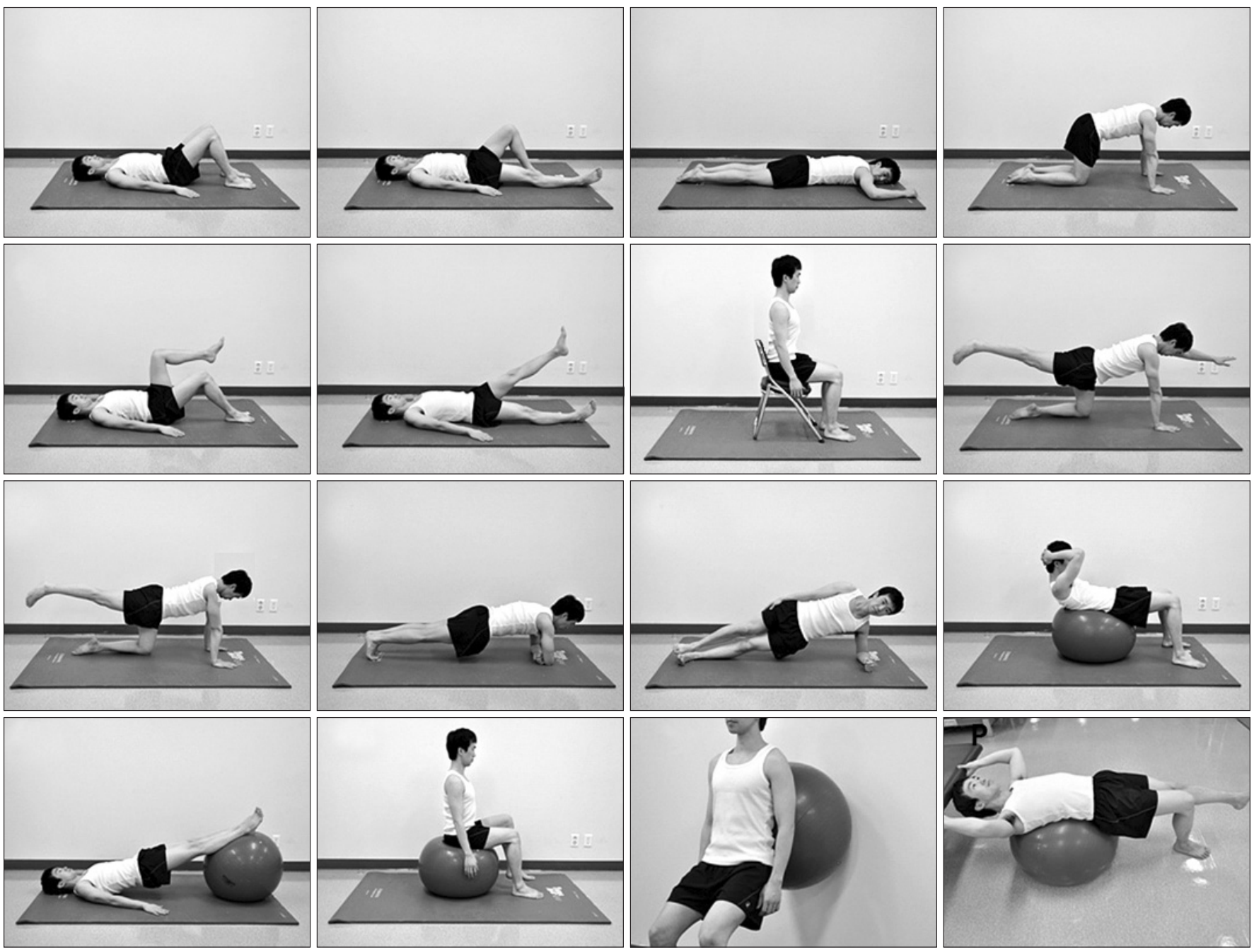

Fig. 1. Lumbar stabilization exercises. 


\section{MATERIALS AND METHODS}

Patients complaining of nonspecific LBP without any structural or neuropsychological cause, for more than 3 months, were recruited from the rehabilitation outpatient-clinic. Exclusion criteria included a history of neurological, infectious, and systemic diseases, including cerebrovascular disease, spinal cord disease, spondylitis, cancer, rheumatologic disorders, and other chronic diseases that cause long-term immobilization. Patients who had undergone prior surgery for back pain, patients who were prescribed exercise therapy in the past, patients who seemed to have radicular pain due to nerve root involvement on physical examination, and patients with structural lesions, such as spondylolisthesis, vertebral bone fracture, scoliosis, and kyphosis on X-ray, were also excluded.

A total of 24 patients were enrolled in the study and randomly assigned to one of the two groups, a lumbar stabilization exercise group ( $\mathrm{n}=12)$ and a conventional lumbar dynamic exercise group $(n=12)$ by a computer-generated random number sequence. Two patients in the lumbar dynamic strengthening exercise group and one patient in the lumbar stabilization exercise group dropped out for personal reasons. The remaining 21 subjects completed the 8-week exercise program without incident.

Each exercise session lasted 60 minutes and was performed 2 days per week, for 8 weeks. All patients in both groups performed warm-up stretching exercises for 15 minutes before the main exercises and cool down exercises for 10 minutes after each session. All exercises were performed in the treatment room under the supervision
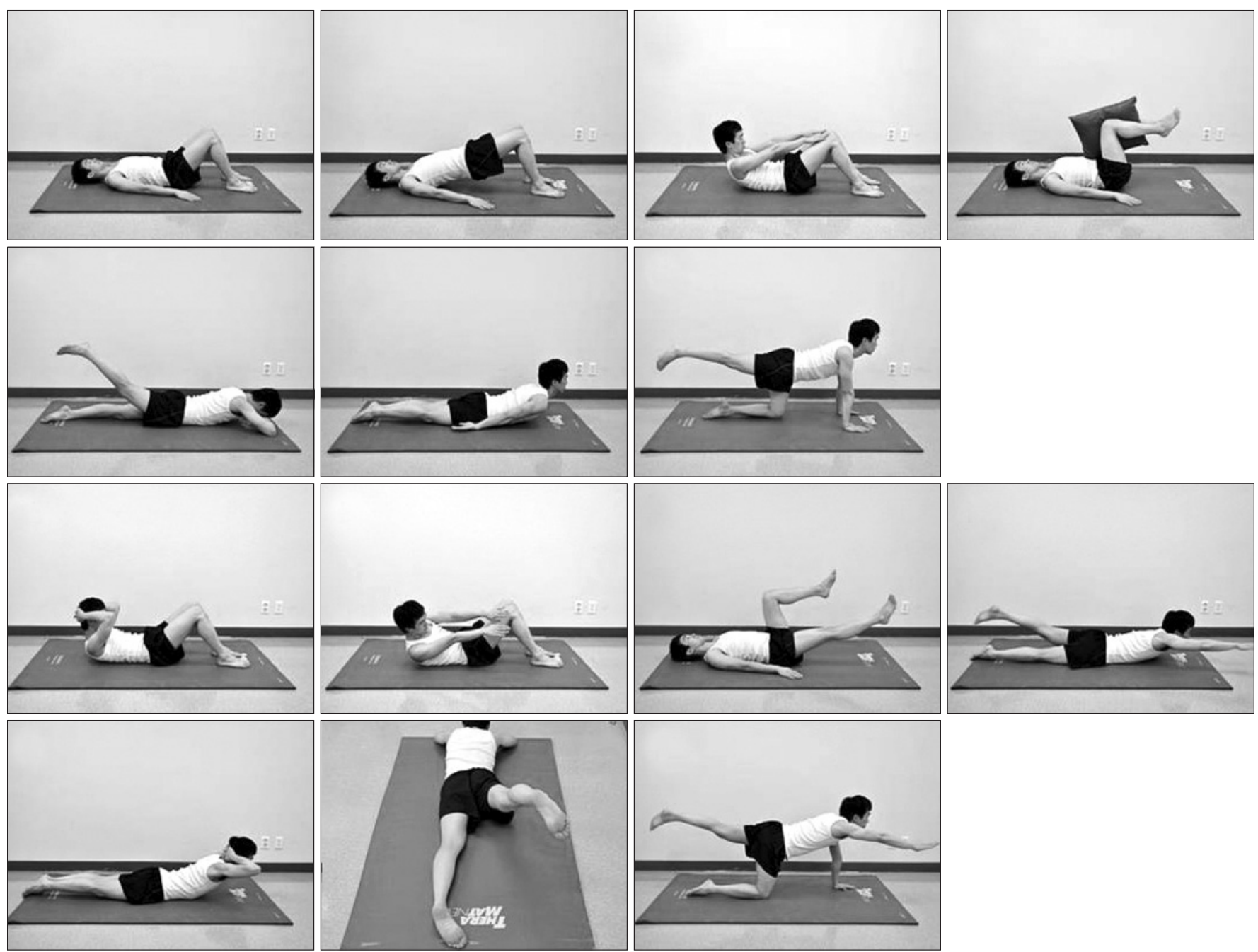

Fig. 2. Lumbar dynamic strengthening exercises. 
of a physical therapist with technical knowledge. The therapist put each patient into the appropriate position to achieve the correct posture and muscle contraction.

Lumbar stabilization exercises consisted of 16 exercises, which were aimed to strengthen the deep lumbar stabilizing muscles: the transversus abdominis, lumbar multifidi, and internal obliques. All 16 stabilization exercises were performed once, consecutively, and in the same order (Fig. 1). Before each exercise, the physical therapist gave detailed verbal explanation and visual instructions (pictures), regarding the start and end positions. All exercises were conducted according to the following specific principles: breathe in and out, gently and slowly draw in your lower abdomen below your umbilicus without moving your upper stomach, back or pelvis" [14]; resulting in a situation referred to as hollowing [15]. Subjects practiced "hollowing" with a therapist providing verbal instruction and tactile feedback until they were able to perform the maneuver in a satisfactory manner. In addition, a "bulging" of the multifidus muscle should have been felt by the therapist when the fingers were placed on either side of the spinous processes of the L4 and L5 vertebrae, directly over the belly of this muscle [14]. These feedback techniques provided by precise palpitation of the appropriate muscles, ensure effective muscle activation [11].

Conventional lumbar dynamic strengthening exercises consisted of 14 exercises (Fig. 2), which activated the extensor (erector spinae) and flexor (rectus abdominis) muscle groups.

For all exercises in both groups, the final static position was held for 10 seconds, and each exercise was performed for 10 repetitions. There was a pause of 3 seconds between repetitions and a 60 -second rest between each exercise. Exercise intensity (holding time and number of repetitions) was increased gradually, based on the tolerance of each patient.

Lumbar extension strength was assessed using a MedX (MedX Holdings Inc., Ocara, FL, USA) lumbar extension machine, which fixes the pelvis, thigh, and knee, to ensure complete stabilization allowing measurements of lumbar extension strength at flexion angles from $0^{\circ}$ to $72^{\circ}$ at intervals of $12^{\circ}\left(0^{\circ}, 12^{\circ}, 24^{\circ}, 36^{\circ}, 48^{\circ}, 60^{\circ}\right.$, and $\left.72^{\circ}\right)$ [16]. Strength tests were conducted at each angle with an approximately 10 seconds rest between tests. Differences in the maximal isometric strength at a given angle of flexion were compared between the groups, before and after the exercise. The percentage change in muscle strength (strength after exercise-strength before exercise/strength before exercise $\times 100 ; \Delta \%$ ) after the dynamic strengthening exercises and lumbar stabilization exercises was also compared.

All evaluations were conducted by an examiner who was not the treating therapist. The examiner was aware of the study design, but was blinded to the group assignments. The examiner and a clinical physical therapist with 10 years of experience in musculoskeletal rehabilitation, who had attended specialized stabilization exercise seminars and was very familiar with the application of these exercise interventions (about 2 years direct experience) before the initiation of the trial, were responsible for supervising all the exercise sessions.

The severity of LBP was evaluated using a visual analog scale (VAS), which ranged from 0 to 100 [17]. The VAS consists of line $(10 \mathrm{~cm}$ in length) with the left extremity indicating "no pain" and the right extremity indicating "unbearable pain." Participants were asked to mark the line to indicate their level of pain. Higher values suggest more intense pain. This instrument shows good reproducibility for assessing pain levels [18]. The patients indicated the average severity of their LBP over the previous 3 days.

The modified Oswestry Low Back Pain Disability Questionnaire (ODQ; also known as the Oswestry Disability Index) was used to evaluate the functional disability and quality of life [19]. This questionnaire was filled out before and after the 8-week exercise program.

This study was approved by the ethics committee of our institute and all subjects provided written informed consent.

\section{Statistical analysis}

Differences in general characteristics (e.g., male/female) between the two groups were analyzed using the Fisher's exact test. Differences in the baseline mean VAS, ODQ scores, and in the strength of the lumbar extensors between the two groups were analyzed using the MannWhitney U-test.

The baseline mean values were compared with the mean values after the treatment within groups using the Wilcoxon's test. The Mann-Whitney U-test was used to compare maximal isometric strength, VAS, and ODQ 
score between the two groups. Statistical significance was defined as a p-value $<0.05$. All statistical analysis were performed using SPSS ver. 18 (SPSS Inc., Chicago, IL, USA).

\section{RESULTS}

All 21 subjects (mean age, $28.5 \pm 4.9$ years) who visited the Department of Rehabilitation outpatient clinic for chronic LBP were enrolled prospectively. There were no significant differences in the general characteristics or the baseline VAS and ODQ scores between the lumbar stabilization exercise and the dynamic lumbar strengthening exercise groups (Table 1).

The baseline maximal isometric contraction strength of

Table 1. Baseline characteristics of the subjects

\begin{tabular}{lcc}
\hline & $\begin{array}{c}\text { Dynamic } \\
\text { strengthening } \\
\text { group }(\mathbf{n}=\mathbf{1 0})\end{array}$ & $\begin{array}{c}\text { Stabilization } \\
\text { exercise group } \\
(\mathbf{n}=\mathbf{1 1})\end{array}$ \\
\hline Age (yr) & $28.6 \pm 4.9$ & $28.4 \pm 5.0$ \\
Sex & & \\
$\quad$ Male & $6(60)$ & $8(72.7)$ \\
\hline Female & $4(40)$ & $3(27.3)$ \\
Body weight (kg) & $68.2 \pm 14.3$ & $67.4 \pm 12.9$ \\
Height (cm) & $172.3 \pm 6.3$ & $171.4 \pm 5.1$ \\
Baseline means (score) & & \\
\hline VAS & $34.2 \pm 17.1$ & $33.5 \pm 18.4$ \\
\hline ODQ & $15.5 \pm 4.3$ & $14.7 \pm 2.9$ \\
\hline
\end{tabular}

Values are presented as mean \pm standard deviation or number (\%).

VAS, visual analog scale; ODQ, modified Oswestry Low Back Pain Disability Questionnaire. the lumbar extensors at all angles of lumbar flexion did not differ significantly between the exercise groups, although the data was not shown.

Compared with that of the baseline, lumbar extension strength at all angles of lumbar flexion improved significantly in both groups after 8 weeks (Table 2). Table 3 shows the difference in maximal isometric contraction strength at a given angle of lumbar flexion before and after the exercise. Improvements were significantly larger in the lumbar stabilization exercise group at $0^{\circ}$ and $12^{\circ}$ of lumbar flexion than in the dynamic strengthening exercise group (Table 3 ). When the percentage change in maximal isometric strength at each angle $(\Delta \%)$ was examined, the improvements were significantly greater in the lumbar stabilization exercise group, at $0^{\circ}$ and $12^{\circ}$ of

Table 3. Comparison of the improvements in lumbar extensor strength at different angles of lumbar flexion in two groups (unit, ft-lb)

\begin{tabular}{cccc}
\hline $\begin{array}{c}\text { Lumbar } \\
\text { flexion } \\
\left({ }^{\circ}\right)\end{array}$ & $\begin{array}{c}\text { Dynamic } \\
\text { strengthening } \\
\text { group }\end{array}$ & $\begin{array}{c}\text { Stabilization } \\
\text { exercise group }\end{array}$ & $\begin{array}{c}\text { p- } \\
\text { value }\end{array}$ \\
\hline$\Delta 0$ & $30.2 \pm 26.9$ & $47.0 \pm 22.5$ & $0.05^{*}$ \\
$\Delta 12$ & $23.0 \pm 21.2$ & $52.8 \pm 26.2$ & $<0.01^{*}$ \\
\hline$\Delta 24$ & $27.0 \pm 22.8$ & $52.7 \pm 28.7$ & 0.07 \\
\hline 36 & $28.9 \pm 18.3$ & $52.7 \pm 26.6$ & 0.09 \\
\hline$\Delta 48$ & $41.4 \pm 25.7$ & $48.5 \pm 25.5$ & 0.71 \\
\hline 660 & $44.9 \pm 25.9$ & $51.5 \pm 27.4$ & 0.82 \\
\hline 42 & $42.4 \pm 28.9$ & $52.1 \pm 31.2$ & 0.66 \\
\hline
\end{tabular}

Values are presented as the mean \pm standard deviation.

$\Delta$, difference in maximal isometric strength before and after exercise at a given angle.

${ }^{*} \mathrm{p}<0.05$.

Table 2. Changes in maximal isometric strength of the lumbar extensors after exercise (unit, ft-lb)

\begin{tabular}{cccccccc}
\hline \multirow{2}{*}{$\begin{array}{c}\text { Lumbar flexion } \\
\left({ }^{\circ}\right)\end{array}$} & \multicolumn{3}{c}{ Dynamic strengthening group } & & \multicolumn{3}{c}{ Stabilization exercise group } \\
\cline { 2 - 3 } \cline { 6 - 8 } & Before exercise & After exercise & p-value* & & Before exercise & After exercise & p-value* \\
\hline 0 & $104.0 \pm 42.5$ & $135.2 \pm 75.9$ & $<0.01$ & & $92.6 \pm 58.0$ & $137.5 \pm 62.4$ & $<0.01$ \\
12 & $132.4 \pm 52.8$ & $155.4 \pm 41.7$ & $<0.01$ & & $115.6 \pm 62.9$ & $168.5 \pm 67.9$ & $<0.01$ \\
\hline 24 & $154.0 \pm 55.7$ & $181.2 \pm 42.3$ & $<0.01$ & & $136.5 \pm 65.1$ & $189.3 \pm 61.3$ & $<0.01$ \\
\hline 36 & $176.8 \pm 61.5$ & $205.7 \pm 68.4$ & $<0.01$ & & $161.5 \pm 65.3$ & $211.2 \pm 67.7$ & $<0.01$ \\
48 & $182.0 \pm 68.5$ & $224.4 \pm 73.1$ & $<0.01$ & & $171.0 \pm 62.4$ & $219.5 \pm 65.3$ & $<0.01$ \\
60 & $201.1 \pm 70.3$ & $246.0 \pm 79.2$ & $<0.01$ & & $182.5 \pm 66.5$ & $234.0 \pm 70.8$ & $<0.01$ \\
\hline 72 & $214.8 \pm 74.5$ & $257.2 \pm 84.7$ & $<0.01$ & & $197.3 \pm 73.0$ & $249.4 \pm 76.6$ & $<0.01$ \\
\hline
\end{tabular}

Values are presented as mean \pm standard deviation.

*Significant difference compared with before exercise, $\mathrm{p}<0.05$. 


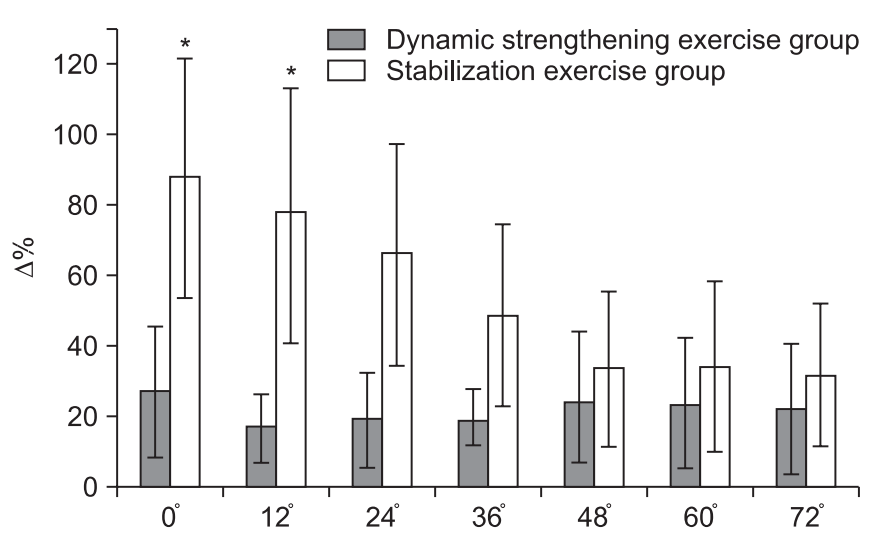

Fig. 3. The percentage change in maximal isometric muscle strength at each angle $(\Delta \%)$ in the lumbar dynamic strengthening exercise and the stabilization exercise groups. The lumbar stabilization exercise group showed significantly greater improvement at $0^{\circ}$ and $12^{\circ}$ than the dynamic strengthening group. *Significant difference between lumbar stabilization exercise and dynamic strengthening exercise group.

lumbar flexion, than in the dynamic strengthening exercise group (Fig. 3).

The VAS reported by all subjects decreased significantly after the treatment, although the difference between the two groups was not significant. However, the ODQ scores significantly improved in the stabilization exercise group (but not in the dynamic strengthening exercise group) compared with the baseline scores. In stabilization exercise group showed better outcome in ODQ score than dynamic lumbar strengthening exercise group, although the difference between these two groups was not significant (Table 4).

\section{DISCUSSION}

The aim of this study was to compare the effects of lumbar stabilization exercises and lumbar dynamic strengthening exercises on the maximal isometric strength of the lumbar extensor muscles, pain severity, and functional disability in patients with chronic LBP. We found that both exercises improved the maximal isometric strength of lumbar extensors and pain severity. However, the functional improvements and lumbar extensor strength at low lumbar flexion angles were both better in the stabilization exercise group than that of the dynamic strengthening exercise group.

Among the abdominal muscles, the transverse ab-
Table 4. Differences in the improvements in VAS and ODQ scores in two groups

\begin{tabular}{lccc}
\hline & $\begin{array}{c}\text { Dynamic } \\
\text { strengthening } \\
\text { group }\end{array}$ & $\begin{array}{c}\text { Stabilization } \\
\text { exercise } \\
\text { group }\end{array}$ & $\begin{array}{c}\text { p- } \\
\text { value }^{\text {a) }}\end{array}$ \\
\hline$\triangle \mathrm{VAS}$ & $14.1 \pm 8.2^{*}$ & $16.7 \pm 7.0^{*}$ & 0.66 \\
$\triangle \mathrm{ODQ}$ & $3.6 \pm 1.5$ & $6.1 \pm 1.9^{*}$ & 0.07 \\
\hline
\end{tabular}

Values are presented as the mean \pm standard deviation. VAS, visual analog scale; ODQ, modified Oswestry Low Back Pain Disability Questionnaire; $\Delta$, difference in the mean values before and after exercise.

${ }^{a)}$ Comparison of the changes in the scores between the lumbar stabilization exercise and dynamic lumbar strengthening exercise groups. *Significant difference compared with before exercise, $\mathrm{p}<0.05$.

dominal, multifidus, and internal oblique muscles help to increase the intra-abdominal pressure, thereby contributing to the spinal and pelvic stability $[18,20,21]$. Our lumbar stabilization exercise group included some lumbar dynamic exercises, which may have strengthened the lumbar extensors at the large lumbar flexion angle in this group of patients. However, functional improvements and lumbar extensor strength at low lumbar flexion angles were both better in the stabilization exercise group, suggesting that these improvements were due to the stabilization exercises. In terms of length-tension relationships, the lumbar extensors are at their shortest at the flexion angles of $0^{\circ}$ and $12^{\circ}$, thereby minimizing the isometric strength [22].

In our study, the improvement of ODQ score did not differ significantly between the lumbar stabilization exercise and dynamic strengthening exercise groups. Generally, ODQ scores of less than 20 indicate that functional disability is not regarded as significant in daily life [23]. Subjects in both of our groups had low ODQ scores and reported no significant difficulties in daily life, despite having chronic LBP. Thus, the degree of improvement after an 8-week program may not be reflected in this outcome. However, the improvement of ODQ scores tended to be higher in the lumbar stabilization exercise group than the dynamic lumbar strengthening exercise group. Moreover, the ODQ scores improved significantly after lumbar stabilization exercise, whereas there was no difference in ODQ scores before and after the dynamic lumbar strengthening exercise. These findings are supported by other studies [11] in which stabilization exercises 
translated into pain and functional capacity improvements.

A multicenter randomized controlled trial by Ferreira et al. [24], compared general exercises and lumbar stabilization exercises in patients with chronic LBP. The lumbar stabilization exercises groups showed marginally better outcomes than the general exercise group after 8 weeks in VAS and score of the Roland Morris Disability Questionnaire [25], but there was no significant difference between the two groups. These results were consistent with our results. This was a high-quality study and clinically relevant, however, the study enrolled a mixed group of subjects (including patients with disc lesions, osteoarthritis, and leg pain), which makes comparison difficult.

The present study has several limitations, including the small number of patients and their relatively young age; therefore, the results cannot be generalized to all patients with nonspecific chronic LBP. Also, the long-term effects of these exercise protocols cannot be predicted; further studies are needed with larger sample sizes and longer follow-up periods. The strength of the deep lumbar muscles was not monitored directly, using a needle electromyography, ultrasound measurements of deep muscle thickening, or muscle activation using pressure biofeedback [26]. Because we measured only the maximal isometric strength of the lumbar extensors, the results may not reflect the overall strength of the deep lumbar stabilization muscles.

In conclusion, both lumbar stabilization and conventional lumbar dynamic strengthening exercises increased the strength of the lumbar extensor muscles and reduced LBP. However, compared to that of lumbar dynamic strengthening exercises, lumbar stabilization exercises were more effective for strengthening lumbar extensors at small lumbar flexion angle and for improving the functional disability in patients with nonspecific chronic LBP.

\section{CONFLICT OF INTEREST}

No potential conflict of interest relevant to this article was reported.

\section{REFERENCES}

1. Rozenberg S. Chronic low back pain: definition and treatment. Rev Prat 2008;58:265-72.
2. Lawrence JP, Greene HS, Grauer JN. Back pain in athletes. J Am Acad Orthop Surg 2006;14:726-35.

3. Davies JE, Gibson T, Tester L. The value of exercises in the treatment of low back pain. Rheumatol Rehabil 1979;18:243-7.

4. Manniche C, Lundberg E, Christensen I, Bentzen L, Hesselsoe G. Intensive dynamic back exercises for chronic low back pain: a clinical trial. Pain 1991;47:5363.

5. McKenzie RA. Prophylaxis in recurrent low back pain. N Z Med J 1979;89:22-3.

6. Plum P, Rehfeld JF. Treatment of acute and chronic back pain with muscular exercises. Ugeskr Laeger 1985;147:1604-11.

7. Martin PR, Rose MJ, Nichols PJ, Russell PL, Hughes IG. Physiotherapy exercises for low back pain: process and clinical outcome. Int Rehabil Med 1986;8:34-8.

8. Mellin G, Jarvikoski A, Verkasalo M. Treatment of patients with chronic low back pain: comparison between rehabilitation centre and outpatient care. Scand J Rehabil Med 1984;16:77-84.

9. Carpenter DM, Nelson BW. Low back strengthening for the prevention and treatment of low back pain. Med Sci Sports Exerc 1999;31:18-24.

10. Dettori JR, Bullock SH, Sutlive TG, Franklin RJ, Patience $\mathrm{T}$. The effects of spinal flexion and extension exercises and their associated postures in patients with acute low back pain. Spine (Phila Pa 1976) 1995;20:2303-12.

11. O'Sullivan PB, Phyty GD, Twomey LT, Allison GT. Evaluation of specific stabilizing exercise in the treatment of chronic low back pain with radiologic diagnosis of spondylolysis or spondylolisthesis. Spine (Phila Pa 1976) 1997;22:2959-67.

12. Standaert CJ, Weinstein SM, Rumpeltes J. Evidenceinformed management of chronic low back pain with lumbar stabilization exercises. Spine J 2008;8:114-20.

13. Panjabi MM. The stabilizing system of the spine. Part I. Function, dysfunction, adaptation, and enhancement. J Spinal Disord 1992;5:383-9.

14. Urquhart DM, Hodges PW. Differential activity of regions of transverses abdominis during trunk rotation. Eur Spine J 2005; 14:393-400.

15. Richardson CA, Jull GA. Muscle control-pain control. What exercises would you prescribe? Man Ther 1995;1:2-10. 
16. Robinson ME, Greene AF, O'Connor P, Graves JE, MacMillan M. Reliability of lumbar isometric torque in patients with chronic low back pain. Phys Ther 1992;72:186-90.

17. Jensen MP, Chen C, Brugger AM. Interpretation of visual analog scale ratings and change scores: a reanalysis of two clinical trials of postoperative pain. J Pain 2003;4:407-14.

18. Richardson CA, Snijders CJ, Hides JA, Damen L, Pas MS, Storm J. The relation between the transversus abdominis muscles, sacroiliac joint mechanics, and low back pain. Spine (Phila Pa 1976) 2002;27:399-405.

19. Fritz JM, Irrgang JJ. A comparison of a modified Oswestry Low Back Pain Disability Questionnaire and the Quebec Back Pain Disability Scale. Phys Ther 2001;81:776-88.

20. Hodges PW, Richardson CA. Contraction of the abdominal muscles associated with movement of the lower limb. Phys Ther 1997;77:132-42.

21. Panjabi M, Abumi K, Duranceau J, Oxland T. Spinal stability and intersegmental muscle forces: a biomechanical model. Spine (Phila Pa 1976) 1989;14:194-
200.

22. Graves JE, Pollock ML, Foster D, Leggett SH, Carpenter DM, Vuoso R, et al. Effect of training frequency and specificity on isometric lumbar extension strength. Spine (Phila Pa 1976) 1990;15:504-9.

23. Sanderson PL, Todd BD, Holt GR, Getty CJ. Compensation, work status, and disability in low back pain patients. Spine (Phila Pa 1976) 1995;20:554-6.

24. Ferreira ML, Ferreira PH, Latimer J, Herbert RD, Hodges PW, Jennings MD, et al. Comparison of general exercise, motor control exercise and spinal manipulative therapy for chronic low back pain: a randomized trial. Pain 2007;131:31-7.

25. Cairns MC, Foster NE, Wright C. Randomized controlled trial of specific spinal stabilization exercises and conventional physiotherapy for recurrent low back pain. Spine (Phila Pa 1976) 2006;31:E670-81.

26. Cynn HS, Oh JS, Kwon OY, Yi CH. Effects of lumbar stabilization using a pressure biofeedback unit on muscle activity and lateral pelvic tilt during hip abduction in sidelying. Arch Phys Med Rehabil 2006;87:1454-8. 